\title{
How to keep attention from straying: Get engaged!
}

\author{
Charles L. Folk, Edward F. Ester, and Kristof Troemel \\ Villanova University, Villanova, Pennsylvania
}

\begin{abstract}
Previous research has suggested that the involuntary allocation of spatial attention to salient, irrelevant stimuli (i.e., attentional capture) is prevented when attention is in a focused state (e.g., Yantis \& Jonides, 1990). Recent work has suggested that although focused attention may be necessary to prevent attentional capture by irrelevant stimuli, it is not sufficient (e.g., Folk, Leber, \& Egeth, 2002). The present experiments provide evidence that attentional engagement, rather than attentional focusing, prevents capture. Observers performed a rapid serial visual presentation task in which they were asked to identify a target letter defined by color. Peripheral distractors that shared the color of the target produced evidence of attentional capture. This effect was completely eliminated, however, when the peripheral distractor was preceded by a central distractor designed to engage attention on the stream. It is concluded that attentional engagement serves to lock out capture by irrelevant, salient stimuli.
\end{abstract}

Limitations on the attentional resources available for human information processing necessitate a mechanism that selectively allocates the available resources to behaviorally relevant stimuli/tasks. In the case of visual information processing, attention allocation has traditionally been characterized in terms of spatial shifts of an attentional "spotlight" that can move independently of the eyes. It is well established that these shifts of spatial attention can be initiated endogenously - according to the observer's goals - or exogenously - in response to salient environmental stimuli (Jonides, 1981). The latter form of attention allocation is referred to as attentional capture.

It is now well established that attentional capture can be driven by dynamic stimulus properties, such as abrupt visual onset and motion (e.g., Franconeri \& Simons, 2003; Theeuwes, 1991; Yantis \& Jonides, 1984), as well as by static properties, such as featural discontinuities in color, orientation, shape, and luminance (Folk, Remington, \& Johnston, 1992; Theeuwes, 1992). There is also evidence that the capture of attention by such properties can be modulated by top-down attentional set. For example, Folk et al. (1992) showed that when observers must search for a visual target defined by a property such as color (e.g., a red letter among white letters), an irrelevant (i.e., uninformative) precue defined by the same property will capture attention, but precues defined by some other property (e.g., abrupt onset) will not. On the basis of these results, Folk et al. (1992) proposed the contingent involuntary orienting hypothesis, according to which the attention allocation system is set so as to allocate attention only to stimuli containing featural properties that the observer knows to be relevant to the task at hand.

There is also evidence that attentional capture is influenced by the observer's state of attentional focus. Yantis and Jonides (1990; see also Theeuwes, 1991) showed that when observers are given reliable information in advance about the location of an impending target, the ability of irrelevant stimuli (such as abrupt onsets) to capture attention is eliminated. On the basis of these results, it has been argued that attentional capture is limited to situations in which attention is in a distributed state. When spatial attention is focused on a particular location, attentional capture is "locked out." However, in recent years, this conclusion has been challenged. For example, Folk, Leber, and Egeth (2002) suggested that focusing attention on a location is not sufficient to prevent attentional capture. Observers were shown a rapid serial visual presentation (RSVP) stream at fixation and were asked to search for and identify a letter of a particular color (e.g., red). On critical trials, a peripheral distractor (PD) consisting of number signs, one of which was either the same color as the target or a different color from the target, appeared simultaneously with one of the letter frames in the series. The results showed that even with attention highly focused on the central stream, PDs matching the color of the central target letter produced a significant decrement in target report. Thus, it would appear that a focused state of spatial attention is not sufficient to prevent capture by irrelevant stimuli that match top-down attentional control settings.

Are there any conditions under which attentional capture is completely locked out? It has been proposed that shifts of spatial attention involve at least three dissociable processes: (1) disengagement from a current location/object, (2) movement to a new location/object, and (3) engagement on the new location/object (Posner, Cohen, \& Rafal, 1982). The notion that these processes are dissociable suggests that it is possible to focus attention on a location or object without

C. L. Folk, charles.folk@villanova.edu 
actually engaging attention (see also Remington \& Folk, 2001). Indeed, the nature of the RSVP task encourages observers to focus attention on the central stream but to withhold attentional engagement until the property signifying the target (e.g., a particular color) appears. In contrast, the studies of Yantis and Jonides (1990) and Theeuwes (1991) encouraged engagement on the precued location because the precue indicated the target location with $100 \%$ certainty, and no other characters (i.e., nontargets) appeared at the cued location. Thus, the apparent lockout of capture in response to the precue may have been due to the engagement, rather than the focusing, of attention. Although Posner et al. (1982) did not provide a precise definition of engagement, our working definition is that engagement involves the opening of a gate between perceptual processing at a particular location/ time and higher level cognitive processes involved in identification, consolidation, and response selection of stimuli appearing at that location. In the following experiment, we explored the possibility that the engagement of attention is what prevents attentional capture.

Previous work has already shown that the engagement of attention on a central stimulus stream can influence the ability to process peripheral stimuli. For example, Joseph, Chun, and Nakayama (1997) found that the ability to report the presence or absence of featural singletons presented in the periphery is severely degraded if the singleton appears soon after the presentation of a target among a rapid stream of nontargets at fixation. However, note that the inability to report the presence/absence of a peripheral feature singleton does not preclude its ability to elicit an involuntary shift of attention. For example, a number of studies have confirmed that attentional capture can be elicited by cues for which there is no subjective awareness (e.g., Ivanoff \& Klein, 2003; McCormick, 1997). Thus, although Joseph et al. reported that observers were unable to identify the presence or absence of a peripheral singleton, it remains possible that these items nonetheless captured attention.

In order to determine whether the act of engaging attention prohibits irrelevant stimuli appearing outside the locus of engagement from capturing attention, we utilized the basic procedure employed by Folk et al. (2002). However, prior to the appearance of the PD, we forced attention to engage on the centrally presented RSVP stream. This was accomplished with a technique recently reported by Folk, Leber, and Egeth (2008). They presented observers with an RSVP stream inside a box at fixation and, at varying intervals prior to the presentation of the target, briefly changed the color of this central box. Even though this change in box color (termed a central distractor, or CD) was irrelevant to the task (i.e., it required no behavioral response), it nonetheless produced a significant decrement in target report when the box changed to the same color as that of the subsequent target. This effect was similar in character to that found in studies of the attentional blink (AB; Chun \& Potter, 1995; Raymond, Shapiro, \& Arnell, 1992). Although there are a number of models of the precise mechanism underlying the $\mathrm{AB}$, all of them assume some sort of attentional selection or engagement of the first target. Thus, Folk et al. (2008) concluded that their CDs were producing the involuntary selection of stimuli appearing at the time of the $\mathrm{CD}$. Consistent with this interpretation, Folk et al. (2008) found that the letter appearing simultaneously with the CD produced significant priming effects (relative to conditions in which the prime letter did not occur simultaneously with the $\mathrm{CD}$ ), suggesting that attention had engaged on the RSVP stream.

A similar manipulation was incorporated into the present study, with the $\mathrm{CD}$ appearing at varying intervals prior to the presentation of the PD. If it is the act of engagement that prevents attentional capture, the effects of the PD should be eliminated when preceded by a CD that is the same color as the target letter.

\section{EXPERIMENT 1}

\section{Method}

Subjects. The subjects were 54 undergraduates at Villanova University who received class credit for their participation in a 50-min experimental session. All subjects had normal or corrected-tonormal visual acuity and normal color vision. Half of the subjects searched for a red target, half for a green target.

Stimuli and Procedure. Each trial consisted of a sequence of 15 stimulus frames presented at fixation, each appearing for $42 \mathrm{msec}$, with an interframe interval of $42 \mathrm{msec}$. Each frame consisted of a letter surrounded by a square (see Figure 1). The square measured $1.5^{\circ}$ in width, and the letters measured $1.2^{\circ}$ in width and $1.3^{\circ}$ in length and, with the exception of one frame, were colored gray. Letters were selected randomly from the English alphabet (except I, $\mathrm{O}, \mathrm{W}$, and $\mathrm{Z}$, which are unusually distinctive) without replacement and were presented in colors randomly selected from yellow, blue, orange, and either red or green (depending on the color of the target). One of the frames in each trial sequence also contained a set of four number signs (\#) appearing $5.2^{\circ}$ of visual angle above, below, to the right of, and to the left of the center of the square.

Three critical frames occurred in each trial sequence (see Figure 1). The first was the CD frame, occurring equally often (across trials) at Positions 2-9 in the letter sequence. When a distractor appeared in the CD frame (note that on one third of the trials, no CD appeared), it consisted of a change in color of the square surrounding the letters. The color of the CD square was either the same color as the subsequent target or a different color than the target (i.e., red

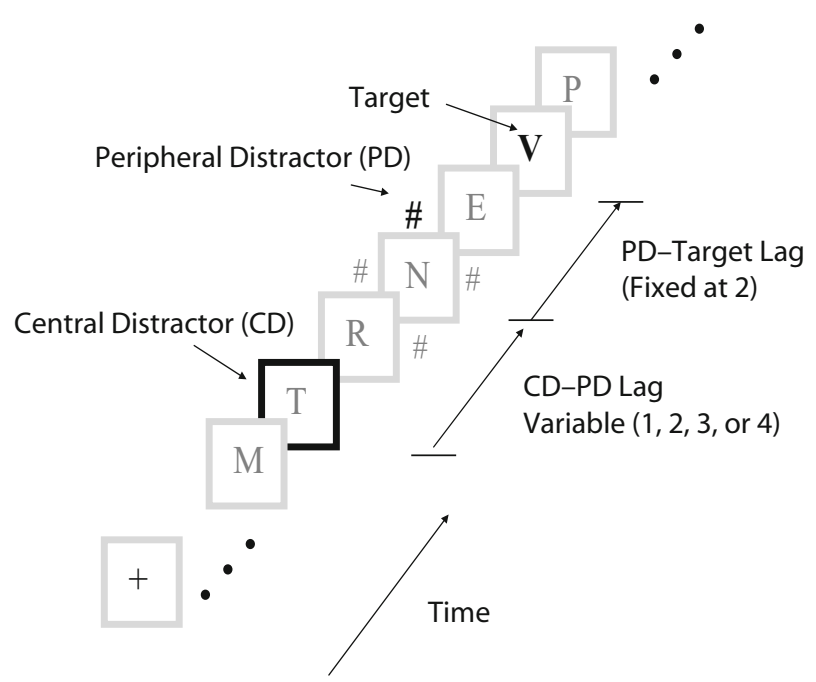

Figure 1. Representation of the stimulus sequence in Experiment 1 . See the text for details on the specific dimensions and colors of the letters and distractors. 
if the target was green or green if the target was red). The second critical frame was the PD frame, which occurred equally often at Positions $6-10$ in the letter sequence. When a PD appeared in the PD frame (note that on one third of the trials, no PD appeared), it consisted of the addition of the four number signs, of which either all were gray or three were gray and one was the color of the target. Finally, the target frame consisted of a red or green letter, depending on the between-subjects condition, surrounded by a gray square. Subjects were explicitly informed to keep their gaze directed at the center of the screen; they were also alerted to the presence of distractors and were instructed to try to ignore them. Subjects identified the target letter by pressing the corresponding key on the keyboard.

Target color (red or green) was varied across subjects, whereas CD type (no $\mathrm{CD}$, same-color $\mathrm{CD}$, different-color $\mathrm{CD}$ ), $\mathrm{PD}$ type (no PD, gray PD, same-color PD), and CD-PD lag $(1,2,3$, or 4) were fully crossed within subjects. The lag between the PD and the target was fixed at two. All combinations of the three within-subjects variables were presented once, in random order, within a block. Subjects were presented with 12 blocks, each of which consisted of 36 trials.

\section{Results}

Mean response accuracy as a function of $\mathrm{CD}$ type, $\mathrm{PD}$ type, CD-PD lag, and target color were entered into a mixed ANOVA with target color as the single between- subjects factor. There was no main effect of target color, nor did it enter into any interactions with any of the other factors. The results, collapsed across target color, are shown in Figure 2. In addition to a significant main effect of CD-PD lag, both PD type and CD type produced significant main effects ( $p<.0001$ for all main effects). As is evident in Figure 2, the main effect of PD type derives from the cost associated with the same-color-PD condition, relative to the no-PD and gray-PD conditions, which did not differ significantly from one another. Similarly, the main effect of $\mathrm{CD}$ type is driven by the same-color-CD condition relative to the no-CD and different-color-CD conditions, which also did not differ significantly from one another. In addition, the effect of same-color CDs varied as a function of CD-PD lag $[F(6,318)=21.49, p<$ $.0001, \eta^{2}=.406$, for the interaction]. The latter pattern is consistent with the same-color $\mathrm{CD}$ producing engagement of attention on the central stream, followed by the gradual disengagement over the course of $600 \mathrm{msec}$.

The critical finding is that the effect of the PD depended on the nature of the $\mathrm{CD}$, yielding a significant $\mathrm{PD}$ type $\times \mathrm{CD}$ type interaction $\left[F(4,212)=14.15, p<.001, \eta^{2}=.268\right]$.
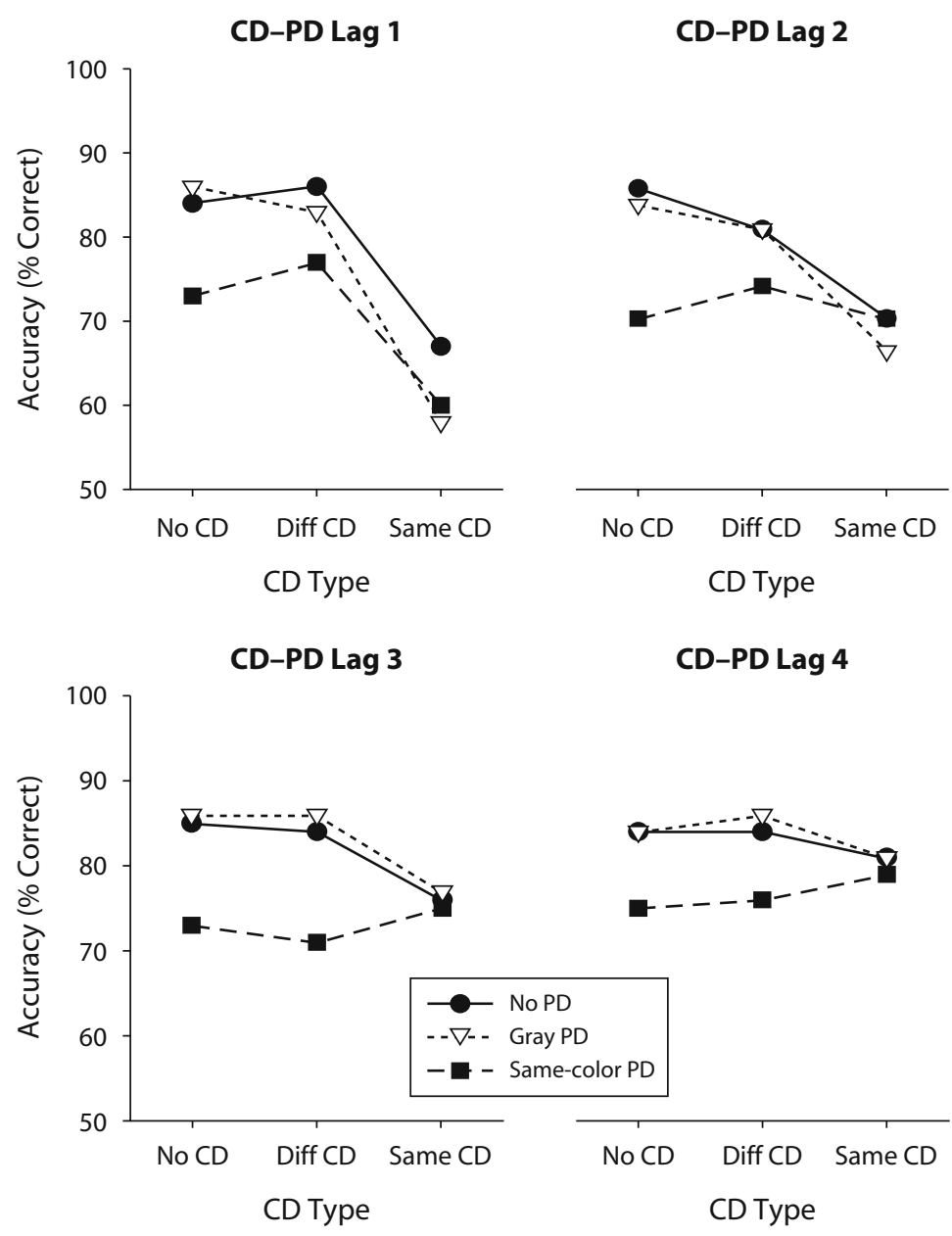

Figure 2. Mean accuracy as a function of central distractor (CD) type, peripheral distractor (PD) type, and CD-PD lag in Experiment 1. Diff CD, different-color CD; Same CD, same-color CD. 
Specifically, as is evident in Figure 2, the decrement in performance associated with same-color PDs was eliminated when the PD was preceded by a same-color $\mathrm{CD}$. The threeway interaction between $\mathrm{CD}$ type, $\mathrm{PD}$ type, and $\mathrm{CD}-\mathrm{PD}$ lag failed to reach significance $[F(12,636)=1.60, p>.08]$.

\section{Discussion}

The fact that same-color PDs produced evidence of attentional capture when the $\mathrm{CD}$ was either absent or gray replicates the results of Folk et al. (2002, Experiment 2) and provides further evidence that attention focused on the central stream is not sufficient to prevent attentional capture when the eliciting PD matches top-down control settings for the central target. Similarly, the fact that a CD the same color as the target produced a decrement in target report relative to an absent or different-color $\mathrm{CD}$ replicates the results of Folk et al. (2008, Experiment 1) and provides evidence that same-color CDs precipitated the engagement of attention on the RSVP stream. Most important, during this engagement period (indexed by the decrement in target report produced by the $\mathrm{CD}$ ), the appearance of a PD produced no further decrement in target report (i.e., over and above that produced by the $\mathrm{CD}$ ), regardless of whether it matched the target color. This pattern is consistent with the notion that engagement of attention by the same-color $\mathrm{CD}$ prevented the capture of attention by the $\mathrm{PD}$, even when the PD matched the target color.

The fact that the interaction between CD type and PD type did not vary significantly with the lag between the $\mathrm{CD}$ and PD may seem surprising, given that the overall effect of the same-color $\mathrm{CD}$ was no longer evident at the longer lags. One might predict that if attention has disengaged by lag 4, same-color PDs should once again capture attention. However, remember that there was a fixed lag of 2 between the PD and the target, which means that a CD-PD lag of 4 translates into a CD-target lag of 6. Thus, although disengagement of attention from the $\mathrm{CD}$ appears to be complete by the time the target arrives 6 lags later, it is likely that attention is still engaged when the $P D$ appears 4 lags after the $C D$. Indeed, the presence of a $C D$ effect at CD-PD lag 2 in Figure 2 (which translates to a $\mathrm{CD}$-target lag of 4) is consistent with this interpretation.

One other reason the lack of a three-way interaction may seem somewhat surprising is that at lag 1 , there is a trend toward an effect of PD type even in the same-color$\mathrm{CD}$ condition. Experiment 2 was conducted, in part, to determine whether this trend is real. In addition, although the data from Experiment 1 are consistent with the hypothesis that attentional engagement can prevent attentional capture, one might argue that conditions may still exist in which irrelevant stimuli can override even attentional engagement. For example, Theeuwes (1991) argued that the relative salience of stimuli can impact their ability to capture attention. Perhaps the color distractors in Experiment 1 were presented so far in the periphery that their salience was insufficient to override the effects of attentional engagement. In Experiment 2, we explored this possibility by directly manipulating the proximity of the distractors to the central stream. For one group of observers, distractors appeared peripherally, as did those in Experiment 1.
In another condition, the distractors appeared nearly contiguous with the frame around the central stream.

\section{EXPERIMENT 2}

\section{Method}

Subjects. Thirty-two undergraduates at Villanova University participated in a 50-min experimental session in exchange for credit toward fulfillment of a class research requirement. All subjects had normal or corrected-to-normal visual acuity and normal color vision. Half of the subjects were randomly assigned to the far-distractor condition and half to the near-distractor condition. For each of these conditions, half were assigned to the red-target condition and half to the green-target condition.

Stimuli, Procedure, and Design. Stimuli were similar to those used in Experiment 1, except that in the near-distractor condition, the inner edge of the number signs making up the distractor stimulus appeared approximately $0.25^{\circ}$ of visual angle from the sides of the central box in which the letter stream appeared. The procedure and design were identical to those used in Experiment 1, with the exception that only CD-PD lags 1 and 3 were used, and the distractor distance manipulation was added as a between-subjects variable.

\section{Results}

The data for 1 subject in the red-target version of the neardistractor condition were not included in the data analysis because of an overall error rate of greater than $80 \%$. The remaining data were subjected to a mixed ANOVA with distractor distance and target color as the between-subjects variables and CD-PD lag, CD type, and PD type as the within-subjects variables. The effect of target color was not significant, nor did it interact with any other variables. The results, collapsed across target color, are presented in Figure 3. As in Experiment 1, the main effects of CD type, PD type, and CD-PD lag were all highly significant $(p<.0001$ for all). Again, the main effect of $C D$ type was driven solely by the costs associated with the same-color-CD relative to the no-CD and different-color-CD conditions. Likewise, the main effect of PD type was driven solely by the costs associated with the same-color-PD relative to the no-PD and gray-PD conditions. The main effect of distractor distance was also significant, with near distractors producing lower overall performance $(63 \%)$ than did far distractors $(76 \%)\left[F(1,29)=7.21, p<.05, \eta^{2}=.249\right]$. The overall effect of distractor distance suggests that we were successful in enhancing the salience of the distractors by moving them closer to the central stream. The key result, however, is that once again the influence of PDs was modulated by the presence and type of $\operatorname{CD}[F(4,116)=9.38, p<.001$, $\eta^{2}=.244$, for the PD $\times$ CD interaction]. Specifically, as is evident in Figure 3, when the CD was either absent or a different color from the target, same-color PDs produced a cost in performance relative to gray or absent PDs. However, when the $\mathrm{CD}$ color matched the target color, PD type no longer had any significant effect on performance. These effects did not vary with lag, since the three-way interaction between $\mathrm{CD}$ type, $\mathrm{PD}$ type, and CD-PD lag was not significant $[F(4,116)=0.84, p>.50]$. Finally, this pattern also did not vary with distractor distance, since the threeway interaction of $\mathrm{CD}$ type, $\mathrm{PD}$ type, and distance was not significant $[F(4,116)=1.72, p>.15]$. 


\section{Far Condition}

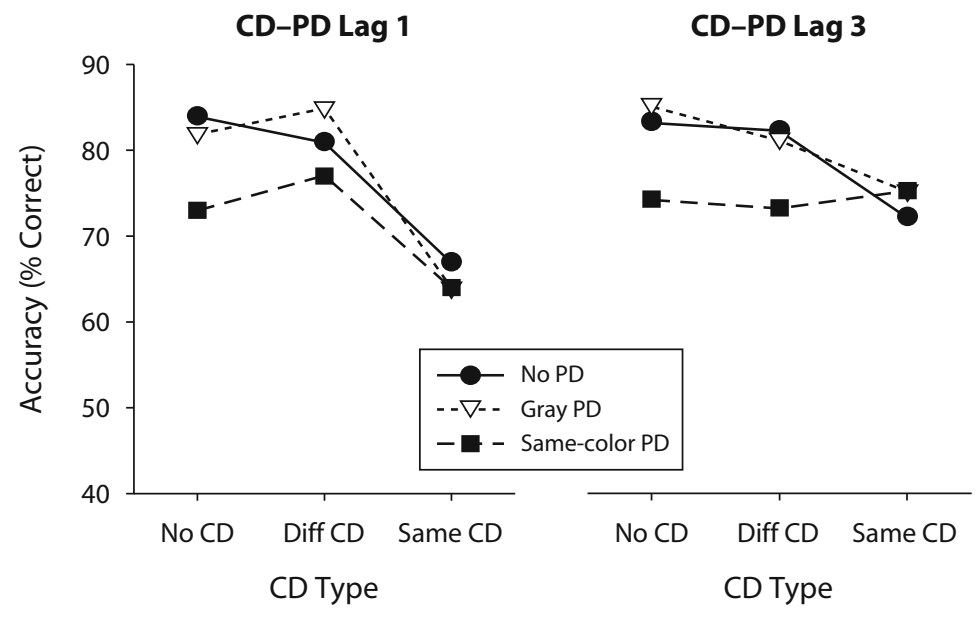

Near Condition

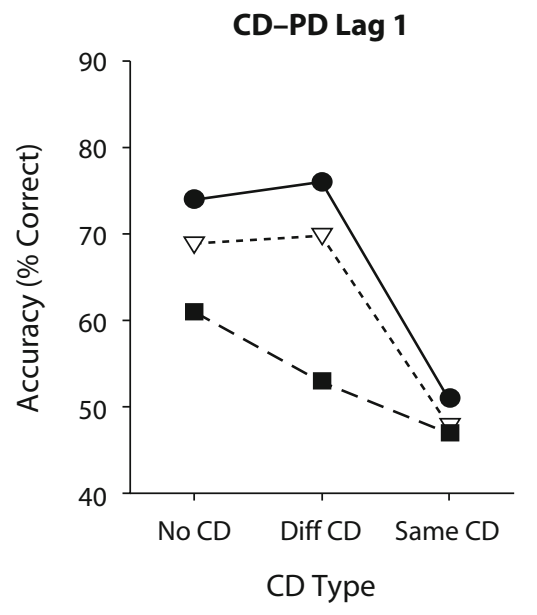

CD-PD Lag 3

Figure 3. Mean accuracy as a function of distractor distance, central distractor (CD) type, peripheral distractor (PD) type, and CD-PD lag in Experiment 2. Diff CD, different-color CD; Same CD, same-color CD.

\section{Discussion}

The results of Experiment 2 replicated those of Experiment 1 and provide further evidence for the hypothesis that the engagement of attention by the $\mathrm{CD}$ prevents the capture of attention. Moreover, the present results show that this pattern is evident even when the distractors are in very close proximity to the central stream, suggesting that increasing the salience of the PDs, at least in terms of their proximity, does not render them capable of overriding the effects of attentional engagement. In addition, there is no hint of the trend toward PD effects in the same-color-CD condition at lag 1, suggesting that the trend observed in Experiment 1 is not real.

\section{GENERAL DISCUSSION}

The present experiments were designed to test the hypothesis that attentional engagement is the necessary condition for the lockout of capture. The results of the present experiments provide clear support for this hypothesis.
First, although attention was presumably focused on the central stream, the presentation of a same-color PD produced a decrement in performance indicative of attentional capture. This result replicates those of Folk et al. (2002), showing that the focusing of attention cannot always prevent capture. Second, CDs whose color matched that of the target also produced a decrement in target report. This result replicates those of Folk et al. (2008) and suggests that same-color CDs produce the involuntary engagement of attention on the central stream. Most important, the capture by same-color PDs (as indexed by the PD-type effect) was eliminated when attention was engaged by the $\mathrm{CD}$ (as indexed by the CD-type effect), even when the salience of distractors was enhanced by presenting them in very close proximity to the central stream. Together, these results suggest that attentional capture by salient peripheral stimuli (even those that match attentional control settings) can be locked out by the engagement of attention.

There are several alternative accounts to consider, however. First, one might argue that the elimination of the PD 
effect following a same-color CD reflects a floor effect. That is, perhaps PDs actually do capture attention even when attention has been engaged by a same-color CD, but the effects of such capture are not evident, because engagement itself has already reduced performance to some absolute minimum level. The CD-PD lag effects observed in both experiments provide strong evidence against this possibility. Specifically, although performance in the same-color-CD condition increased with CD-PD lag, PD type still had no significant effect. Consider, for example, lags 1 and 3 of Experiment 1 . When the color of the CD was the same as that of the target, overall performance was $66 \%$ at lag 1 and increased to $79 \%$ at lag 3 . This suggests that at lag 3 , there would be room for further decrements in performance associated with the presence of PDs. However, there was no effect of PD type at either lag.

Second, one might argue that same-color CDs result in a tighter focus of attention, as opposed to attentional engagement. If so, this would suggest that attentional focus is sufficient to prevent capture and that the prevention of capture is a matter of the degree of attentional focus. This possibility is also unlikely, however, because if CDs produce a tighter attentional focus, target identification would be enhanced rather than degraded, because more attentional resources would be focused on the central stream (see Folk et al., 2008, for a full discussion of this point). The fact that performance declines in the presence of a same-color CD is uniquely consistent with the involuntary engagement of attention on the stream, which presumably results in processing of nontarget characters, which, in turn, produces a decrement in target report similar to that found in studies of the AB (e.g., Chun \& Potter, 1995).

Although the present results show that the capture of attention by PDs is prevented by attentional engagement, several studies suggest that the effects of attentional engagement may be different for distractors that appear within the focus of attention. For example, using a traditional $\mathrm{AB}$ paradigm in which subjects must report two targets (T1 and T2) in the stream, Nieuwenstein, Chun, van der Lubbe, and Hooge (2005) found that nontargets appearing within the stream that shared the color of T2 reduced the magnitude of the $\mathrm{AB}$ produced by $\mathrm{T} 1$. In contrast, Wee and Chua (2004) found that CDs appearing between $\mathrm{T} 1$ and $\mathrm{T} 2$ increased the duration of the $\mathrm{AB}$ produced by $\mathrm{T} 1$. The results of these studies suggest that distractors within the focus of attention can influence performance even when attention has been previously engaged. Thus, the rules of attentional capture may be different for stimuli that appear within, rather than outside, the focus of spatial attention.

Finally, note that although the present results suggest that attentional engagement of the central stream prevents the capture of attention by PDs, the functional mechanisms underlying this effect remain to be determined. That is, we have assumed that the act of engagement itself (i.e., the opening of a gate between perceptual processes and higher level cognitive processes) prevents the capture of spatial attention. However, it is also possible that the consequences of opening the gate (e.g., the initiation of selection, encoding, consolidation, response selection, etc.) also play a role. Thus, future research is needed in order to determine exactly what functional aspects of attentional engagement result in the lockout of attentional capture.

\section{AUTHOR NOTE}

Portions of this research were presented at the Annual Meeting of the Psychonomic Society, Vancouver, November 8, 2003. This research was supported in part by National Science Foundation Grant BCS-9817673 to C.L.F. E.F.E is now at the University of Oregon. Correspondence concerning this article should be sent to C. L. Folk, Department of Psychology, Villanova University, Villanova, PA 19085 (e-mail: charles.folk@villanova .edu).

\section{REFERENCES}

Chun, M. M., \& Potter, M. C. (1995). A two-stage model for multiple target detection in rapid serial visual presentation. Journal of Experimental Psychology: Human Perception \& Performance, 21, 109-127.

Folk, C. L., Leber, A. B., \& Egeth, H. E. (2002). Made you blink! Contingent attentional capture produces a spatial blink. Perception \& Psychophysics, 64, 741-753.

Folk, C. L., Leber, A. B., \& Egeth, H. E. (2008). Top-down control settings and the attentional blink: Evidence for non-spatial contingent capture. Visual Cognition, 16, 616-642.

Folk, C. L., Remington, R. W., \& Johnston, J. C. (1992). Involuntary covert orienting is contingent on attentional control settings. Journal of Experimental Psychology: Human Perception \& Performance, 18, 1030-1044.

Franconeri, S. L., \& Simons, D. J. (2003). Moving and looming stimuli capture attention. Perception \& Psychophysics, 65, 999-1010.

IVANOFF, J., \& KLEIN, R. M. (2003). Orienting of attention without awareness is affected by measurement-induced attentional control settings. Journal of Vision, 3, 32-40.

JONIDES, J. (1981). Voluntary versus automatic control over the mind's eye's movements. In J. B. Long \& A. D. Baddeley (Eds.), Attention and performance IX (pp. 187-203). Hillsdale, NJ: Erlbaum.

Joseph, J. S., Chun, M. M., \& NaKayama, K. (1997). Attentional requirements in a "preattentive" feature search task. Nature, 387, 805-807.

McCormick, P. A. (1997). Orienting attention without awareness. Journal of Experimental Psychology: Human Perception \& Performance, 23, 168-180.

Nieuwenstein, M. R., Chun, M. M., van der Lubbe, R. H. J., \& Hooge, I. T. C. (2005). Delayed attentional engagement in the attentional blink. Journal of Experimental Psychology: Human Perception $\&$ Performance, 31, 1463-1475.

Posner, M. I., Cohen, Y., \& Rafal, R. D. (1982). Neural systems control of spatial orienting. Philosophical Transactions of the Royal Society $B, 298,187-198$

Raymond, J. E., Shapiro, K. L., \& Arnell, K. M. (1992). Temporary suppression of visual processing in an RSVP task: An attentional blink? Journal of Experimental Psychology: Human Perception \& Performance, 18, 849-860.

Remington, R. W., \& Folk, C. L. (2001). A dissociation between attention and selection. Psychological Science, 12, 511-515.

TheEUwes, J. (1991). Exogenous and endogenous control of attention: The effect of visual onsets and offsets. Perception \& Psychophysics, 49, 83-90.

TheEuwes, J. (1992). Perceptual selectivity for color and form. Perception \& Psychophysics, 51, 599-606.

WeE, S., \& ChuA, F. K. (2004). Capturing attention when attention "blinks." Journal of Experimental Psychology: Human Perception \& Performance, 30, 598-612.

YANTIS, S., \& JonIDES, J. (1984). Abrupt visual onsets and selective attention: Evidence from visual search. Journal of Experimental Psychology: Human Perception \& Performance, 10, 601-621.

YAnTIS, S., \& Jonides, J. (1990). Abrupt visual onsets and selective attention: Voluntary versus automatic allocation. Journal of Experimental Psychology: Human Perception \& Performance, 16, 121-134.

(Manuscript received January 10, 2008; revision accepted for publication September 5, 2008.) 